\title{
Bidual Spaces and Reflexivity of Real Normed Spaces $]^{\Uparrow}$
}

\author{
Keiko Narita \\ Hirosaki-city \\ Aomori, Japan
}

\author{
Noboru Endou \\ Gifu National College of Technology \\ Gifu, Japan
}

\author{
Yasunari Shidama \\ Shinshu University \\ Nagano, Japan
}

\begin{abstract}
Summary. In this article, we considered bidual spaces and reflexivity of real normed spaces. At first we proved some corollaries applying Hahn-Banach theorem and showed related theorems. In the second section, we proved the norm of dual spaces and defined the natural mapping, from real normed spaces to bidual spaces. We also proved some properties of this mapping. Next, we defined real normed space of $\mathbb{R}$, real number spaces as real normed spaces and proved related theorems. We can regard linear functionals as linear operators by this definition. Accordingly we proved Uniform Boundedness Theorem for linear functionals using the theorem (5) from 21. Finally, we defined reflexivity of real normed spaces and proved some theorems about isomorphism of linear operators. Using them, we proved some properties about reflexivity. These formalizations are based on [19], 20], 8] and [1].
\end{abstract}

\section{MSC: 46B10 46A25 03B35}

Keywords: continuous dual space; topological duality; reflexivity

MML identifier: DUALSP02, version: 8.1.03 5.26.1224

The notation and terminology used in this paper have been introduced in the following articles: [2], [14], [7], [3], [4], [16], [22], [24], [15], [18], [13], [5], [10], [29], [25], [26], [11], [28], [12], and [6].

\footnotetext{
${ }^{1}$ This work was supported by JSPS KAKENHI 22300285 and 23500029. 


\section{The Applichtion of Hahn-Banach Theorem}

Now we state the propositions:

(1) Let us consider a real normed space $V$, a real normed subspace $X$ of $V$, a point $x_{0}$ of $V$, and a real number $d$. Suppose there exists a non empty subset $Z$ of $\mathbb{R}$ such that $Z=\left\{\left\|x-x_{0}\right\|\right.$, where $x$ is a point of $\left.V: x \in X\right\}$ and $d=\inf Z>0$. Then

(i) $x_{0} \notin X$, and

(ii) there exists a point $G$ of $\operatorname{DualSp}(V)$ such that for every point $x$ of $V$ such that $x \in X$ holds (Bound2Lipschitz $(G, V))(x)=0$ and $(\operatorname{Bound} 2 \operatorname{Lipschitz}(G, V))\left(x_{0}\right)=1$ and $\|G\|=\frac{1}{d}$.

Proof: Consider $Z$ being a non empty subset of $\mathbb{R}$ such that $Z=\{\| x-$ $x_{0} \|$, where $x$ is a point of $\left.V: x \in X\right\}$ and $d=\inf Z>0$. Set $M_{0}=$ $\left\{z+a \cdot x_{0}\right.$, where $z$ is a point of $V, a$ is a real number : $\left.z \in X\right\}$. Set $M=$ NLin $M_{0} . M_{0}$ is linearly closed by [25, (20), (21)]. For every point $v$ of $M$, there exists a point $x$ of $V$ and there exists a real number $a$ such that $v=x+a \cdot x_{0}$ and $x \in X$ by [13, (31)]. Reconsider $r_{0}=0$ as a real number. For every extended real $r$ such that $r \in Z$ holds $r_{0} \leqslant r$. For every points $x_{1}, x_{2}$ of $V$ and for every real numbers $a_{1}, a_{2}$ such that $x_{1}, x_{2} \in X$ and $x_{1}+a_{1} \cdot x_{0}=x_{2}+a_{2} \cdot x_{0}$ holds $x_{1}=x_{2}$ and $a_{1}=a_{2}$ by [26, (5), (35), (15)]. Define $\mathcal{P}$ [object, object] $\equiv$ there exists a point $z$ of $V$ and there exists a real number $a$ such that $z \in X$ and $\$_{1}=z+a \cdot x_{0}$ and $\$_{2}=a$. For every element $v$ of $M$, there exists an element $a$ of $\mathbb{R}$ such that $\mathcal{P}[v, a]$. Consider $f$ being a function from $M$ into $\mathbb{R}$ such that for every element $x$ of $M$, $\mathcal{P}[x, f(x)]$ from [4, Sch. 3]. For every point $v$ of $M$ and for every point $z$ of $V$ and for every real number $a$ such that $z \in X$ and $v=z+a \cdot x_{0}$ holds $f(v)=a$. $f$ is a linear functional in $M$ by [13, (28)], [25, (20), (21)]. For every point $v$ of $M,|f(v)| \leqslant \frac{1}{d} \cdot\|v\|$ by [17, (2)], [18, (2)], [26, (30), (25)]. Reconsider $F=f$ as a point of $\operatorname{DualSp}(M)$. Consider $g$ being a Lipschitzian linear functional in $V, G$ being a point of $\operatorname{DualSp}(V)$ such that $g=G$ and $g\lceil$ (the carrier of $M)=f$ and $\|G\|=\|F\|$. For every point $x$ of $V$ such that $x \in X$ holds (Bound2Lipschitz $(G, V))(x)=0$ by 26 , (10)], [3, (49)].

(2) Let us consider a real normed space $V$, a non empty subset $Y$ of $V$, and a point $x_{0}$ of $V$. Suppose $Y$ is linearly closed and closed and $x_{0} \notin Y$. Then there exists a point $G$ of $\operatorname{DualSp}(V)$ such that

(i) for every point $x$ of $V$ such that $x \in Y$ holds

(Bound2Lipschitz $(G, V))(x)=0$, and 
(ii) $($ Bound2Lipschitz $(G, V))\left(x_{0}\right)=1$.

Proof: Set $X=\operatorname{NLin} Y$. Set $Z=\left\{\left\|x-x_{0}\right\|\right.$, where $x$ is a point of $V: x \in$ $X\}$. Reconsider $r_{0}=0$ as a real number. For every extended real $r$ such that $r \in Z$ holds $r_{0} \leqslant r$. Reconsider $d=\inf Z$ as a real number. $d>0$ by [9, (16), (7)], [18, (7)]. Consider $G$ being a point of $\operatorname{DualSp}(V)$ such that for every point $x$ of $V$ such that $x \in X$ holds (Bound2Lipschitz $(G, V))(x)=0$ and $(\operatorname{Bound} 2 \operatorname{Lipschitz}(G, V))\left(x_{0}\right)=1$ and $\|G\|=\frac{1}{d}$.

Let us consider a real normed space $V$ and a point $x_{0}$ of $V$.

Let us assume that $x_{0} \neq 0_{V}$. Now we state the propositions:

(3) There exists a point $G$ of $\operatorname{DualSp}(V)$ such that

(i) $(\operatorname{Bound} 2 \operatorname{Lipschitz}(G, V))\left(x_{0}\right)=1$, and

(ii) $\|G\|=\frac{1}{\left\|x_{0}\right\|}$.

Proof: Set $X=\mathrm{NLin}\left\{0_{V}\right\}$. Set $Y=$ the carrier of $\operatorname{Lin}\left(\left\{0_{V}\right\}\right)$. For every object $s, s \in Y$ iff $s \in\left\{0_{V}\right\}$ by [27, (8)]. Set $Z=\left\{\left\|x-x_{0}\right\|\right.$, where $x$ is a point of $V: x \in X\}$. For every object $s, s \in Z$ iff $s \in\left\{\left\|x_{0}\right\|\right\}$ by [18, (2)]. Reconsider $d=\inf Z$ as a real number. Consider $G$ being a point of $\operatorname{DualSp}(V)$ such that for every point $x$ of $V$ such that $x \in X$ holds

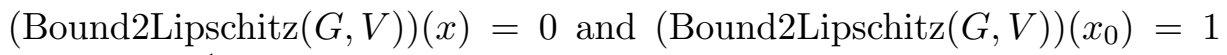
and $\|G\|=\frac{1}{d}$.

(4) There exists a point $F$ of $\operatorname{DualSp}(V)$ such that

(i) $\|F\|=1$, and

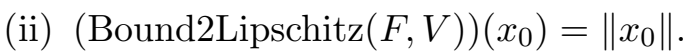

The theorem is a consequence of (3).

Let us consider a real normed space $V$.

Let us assume that $V$ is not trivial. Now we state the propositions:

(5) There exists a point $F$ of $\operatorname{DualSp}(V)$ such that $\|F\|=1$. The theorem is a consequence of (4).

(6) $\operatorname{DualSp}(V)$ is not trivial. The theorem is a consequence of (5).

\section{Bidual Spaces of Real Normed Spaces}

Let us consider a real normed space $V$ and a point $x$ of $V$. Now we state the propositions:

(7) Suppose $V$ is not trivial. Then

(i) there exists a non empty subset $X$ of $\mathbb{R}$ such that $X=\{|(\operatorname{Bound} 2 \operatorname{Lipschitz}(F, V))(x)|$, where $F$ is a point of $\operatorname{DualSp}(V)$ : $\|F\|=1\}$ and $\|x\|=\sup X$, and 
(ii) there exists a non empty subset $Y$ of $\mathbb{R}$ such that $Y=\{|(\operatorname{Bound} 2 \operatorname{Lipschitz}(F, V))(x)|$, where $F$ is a point of $\operatorname{DualSp}(V)$ : $\|F\| \leqslant 1\}$ and $\|x\|=\sup Y$.

The theorem is a consequence of (5) and (4).

(8) If for every Lipschitzian linear functional $f$ in $V, f(x)=0$, then $x=0_{V}$. The theorem is a consequence of (3).

Let $X$ be a real normed space and $x$ be a point of $X$. The functor Bidual $x$ yielding a point of DualSp $(\operatorname{DualSp}(X))$ is defined by

(Def. 1) for every point $f$ of $\operatorname{DualSp}(X)$, it $(f)=f(x)$.

The functor BidualFunc $X$ yielding a function from $X$ into DualSp$(\operatorname{DualSp}(X))$ is defined by

(Def. 2) for every point $x$ of $X$, it $(x)=\operatorname{Bidual} x$.

Let us observe that BidualFunc $X$ is additive and homogeneous and Bidualfunc $X$ is one-to-one.

Let us consider a real normed space $X$.

Let us assume that $X$ is not trivial. Now we state the propositions:

(9) (i) BidualFunc $X$ is a linear operator from $X$ into $\operatorname{DualSp}(\operatorname{DualSp}(X))$, and

(ii) for every point $x$ of $X,\|x\|=\|($ BidualFunc $X)(x) \|$.

(10) There exists a real normed subspace $D$ of $\operatorname{DualSp}(\operatorname{DualSp}(X))$ and there exists a Lipschitzian linear operator $L$ from $X$ into $D$ such that $L$ is bijective and $D=\Im($ BidualFunc $X)$ and for every point $x$ of $X, L(x)=$ Bidual $x$ and for every point $x$ of $X,\|x\|=\|L(x)\|$.

Proof: Set $F=$ BidualFunc $X$. Set $V_{1}=\operatorname{rng} F$. $V_{1} \neq \emptyset$ by [29, (42)]. Reconsider $L=$ BidualFunc $X$ as a function from $X$ into $\Im(F)$. $L$ is additive by [13, (28)]. $L$ is homogeneous by [13, (28)]. For every point $x$ of $X$, $\|x\|=\|L(x)\|$ by [13, (28)].

\section{Uniform Boundedness Theorem for Linear Functionals}

The real normed space of $\mathbb{R}$ yielding a real normed space is defined by the term

(Def. 3) $\left\langle\mathbb{R}, 0(\in \mathbb{R}),+_{\mathbb{R}}, \cdot \mathbb{R},|\square|_{\mathbb{R}}\right\rangle$.

Now we state the proposition:

(11) Let us consider a real normed space $X$, an element $x$ of $\mathbb{R}$, and a point $v$ of the real normed space of $\mathbb{R}$. If $x=v$, then $-x=-v$. 
Let us consider a real normed space $X$ and an object $x$. Now we state the propositions:

(12) $x$ is an additive, homogeneous function from $X$ into $\mathbb{R}$ if and only if $x$ is an additive, homogeneous function from $X$ into the real normed space of $\mathbb{R}$.

(13) $x$ is a Lipschitzian, additive, homogeneous function from $X$ into $\mathbb{R}$ if and only if $x$ is a Lipschitzian, additive, homogeneous function from $X$ into the real normed space of $\mathbb{R}$. The theorem is a consequence of (12).

Now we state the propositions:

(14) Let us consider a real normed space $X$. Then the carrier of $\operatorname{DualSp}(X)=$ the carrier of the real norm space of bounded linear operators from $X$ into the real normed space of $\mathbb{R}$. The theorem is a consequence of (13).

(15) Let us consider a real normed space $X$, points $x, y$ of $\operatorname{DualSp}(X)$, and points $v, w$ of the real norm space of bounded linear operators from $X$ into the real normed space of $\mathbb{R}$. If $x=v$ and $y=w$, then $x+y=v+w$. ProOF: Reconsider $z=x+y$ as a point of $\operatorname{DualSp}(X)$. Reconsider $u=$ $v+w$ as a point of the real norm space of bounded linear operators from $X$ into the real normed space of $\mathbb{R}$. For every object $t$ such that $t \in \operatorname{dom} z$ holds $z(t)=u(t)$ by [14, (29)], [22, (35)].

(16) Let us consider a real normed space $X$, an element $a$ of $\mathbb{R}$, a point $x$ of $\operatorname{DualSp}(X)$, and a point $v$ of the real norm space of bounded linear operators from $X$ into the real normed space of $\mathbb{R}$. If $x=v$, then $a \cdot x=a \cdot v$. Proof: Reconsider $z=a \cdot x$ as a point of $\operatorname{DualSp}(X)$. Reconsider $u=a \cdot v$ as a point of the real norm space of bounded linear operators from $X$ into the real normed space of $\mathbb{R}$. For every object $t$ such that $t \in \operatorname{dom} z$ holds $z(t)=u(t)$ by [14, (30)], [22, (36)].

Let us consider a real normed space $X$, a point $x$ of $\operatorname{DualSp}(X)$, and a point $v$ of the real norm space of bounded linear operators from $X$ into the real normed space of $\mathbb{R}$.

Let us assume that $x=v$. Now we state the propositions:

(17) $-x=-v$. The theorem is a consequence of (16).

(18) $\|x\|=\|v\|$.

Now we state the propositions:

(19) Let us consider a real normed space $X$, and a subset $L$ of $X$. Suppose $X$ is not trivial and for every point $f$ of $\operatorname{DualSp}(X)$, there exists a real number $K_{1}$ such that $0 \leqslant K_{1}$ and for every point $x$ of $X$ such that $x \in L$ holds $|f(x)| \leqslant K_{1}$. Then there exists a real number $M$ such that

(i) $0 \leqslant M$, and 
(ii) for every point $x$ of $X$ such that $x \in L$ holds $\|x\| \leqslant M$.

The theorem is a consequence of (14) and (18).

(20) Let us consider a real normed space $X$, and a non empty subset $L$ of $X$. Suppose $X$ is not trivial and for every point $f$ of $\operatorname{DualSp}(X)$, there exists a subset $Y_{1}$ of $\mathbb{R}$ such that $Y_{1}=\{|f(x)|$, where $x$ is a point of $X: x \in L\}$ and $\sup Y_{1}<+\infty$. Then there exists a subset $Y$ of $\mathbb{R}$ such that

(i) $Y=\{\|x\|$, where $x$ is a point of $X: x \in L\}$, and

(ii) $\sup Y<+\infty$.

Proof: For every point $f$ of $\operatorname{DualSp}(X)$, there exists a real number $K_{1}$ such that $0 \leqslant K_{1}$ and for every point $x$ of $X$ such that $x \in L$ holds $|f(x)| \leqslant K_{1}$ by [2, (46)]. Consider $M$ being a real number such that $0 \leqslant M$ and for every point $x$ of $X$ such that $x \in L$ holds $\|x\| \leqslant M$. Consider $x_{0}$ being an object such that $x_{0} \in L$. Set $Y=\{\|x\|$, where $x$ is a point of $X: x \in L\} . Y \subseteq \mathbb{R}$. For every extended real $r$ such that $r \in Y$ holds $r \leqslant M$.

\section{Reflexivity of Real Normed Spaces}

Let $X$ be a real normed space. We say that $X$ is reflexive if and only if (Def. 4) BidualFunc $X$ is onto.

Let us consider a real normed space $X$. Now we state the propositions:

(21) $X$ is reflexive if and only if for every point $f$ of $\operatorname{DualSp}(\operatorname{DualSp}(X))$, there exists a point $x$ of $X$ such that for every point $g$ of $\operatorname{DualSp}(X)$, $f(g)=g(x)$.

(22) $X$ is reflexive if and only if $\Im(\operatorname{BidualFunc} X)=\operatorname{DualSp}(\operatorname{DualSp}(X))$.

(23) If $X$ is non trivial and reflexive, then $X$ is a real Banach space.

Proof: For every sequence $s_{1}$ of $X$ such that $s_{1}$ is Cauchy sequence by norm holds $s_{1}$ is convergent by [23, (8)], [3, (13)], [26, (16)], [4, (113)].

Now we state the propositions:

(24) Let us consider a real Banach space $X$, and a non empty subset $M$ of $X$. Suppose $X$ is reflexive and $M$ is linearly closed and closed. Then NLin $M$ is reflexive.

Proof: Set $M_{0}=\operatorname{NLin} M$. For every point $y$ of DualSp $\left(\operatorname{DualSp}\left(M_{0}\right)\right)$, there exists a point $x$ of $M_{0}$ such that for every point $g$ of $\operatorname{DualSp}\left(M_{0}\right)$, $y(g)=g(x)$ by [4, (32)], [13, (28)], [3, (49)], [14, (26), (29), (30)].

(25) Let us consider real normed spaces $X, Y$, a Lipschitzian linear operator $L$ from $X$ into $Y$, and a Lipschitzian linear functional $y$ in $Y$. Then $y \cdot L$ is a Lipschitzian linear functional in $X$. 
Proof: Consider $M$ being a real number such that $0 \leqslant M$ and for every vector $x$ of $X,\|L(x)\| \leqslant M \cdot\|x\|$. Set $x=y \cdot L$. For every vectors $v, w$ of $X, x(v+w)=x(v)+x(w)$ by [3, (13)]. For every vector $v$ of $X$ and for every real number $r, x(r \cdot v)=r \cdot x(v)$ by [3, (13)]. Consider $N$ being a real number such that $0 \leqslant N$ and for every vector $v$ of $Y,|y(v)| \leqslant N \cdot\|v\|$. For every vector $v$ of $X,|x(v)| \leqslant M \cdot N \cdot\|v\|$ by [3, (13)].

(26) Let us consider real normed spaces $X, Y$, and a Lipschitzian linear operator $L$ from $X$ into $Y$. Suppose $L$ is isomorphism. Then there exists a Lipschitzian linear operator $T$ from $\operatorname{DualSp}(X)$ into $\operatorname{DualSp}(Y)$ such that

(i) $T$ is isomorphism, and

(ii) for every point $x$ of $\operatorname{DualSp}(X), T(x)=x \cdot L^{-1}$.

Proof: Consider $K$ being a Lipschitzian linear operator from $Y$ into $X$ such that $K=L^{-1}$ and $K$ is isomorphism. Define $\mathcal{P}$ [function, function] $\equiv$ $\$_{2}=\$_{1} \cdot K$. For every element $x$ of $\operatorname{DualSp}(X)$, there exists an element $y$ of $\operatorname{DualSp}(Y)$ such that $\mathcal{P}[x, y]$. Consider $T$ being a function from $\operatorname{DualSp}(X)$ into $\operatorname{DualSp}(Y)$ such that for every element $x$ of $\operatorname{DualSp}(X), \mathcal{P}[x, T(x)]$ from [4, Sch. 3]. For every points $v, w$ of $\operatorname{DualSp}(X), T(v+w)=T(v)+$ $T(w)$ by [3, (13)], [14, (29)]. For every point $v$ of $\operatorname{DualSp}(X)$ and for every real number $r, T(r \cdot v)=r \cdot T(v)$ by [3, (13)], [14, (30)]. For every object $v$ such that $v \in$ the carrier of $\operatorname{DualSp}(Y)$ there exists an object $s$ such that $s \in$ the carrier of $\operatorname{DualSp}(X)$ and $v=T(s)$ by (25), [29, (36)], [3. (39)], [29, (51)]. For every point $v$ of $\operatorname{DualSp}(X),\|T(v)\|=\|v\|$ by [3, (34), (13)], [14, (23)]. For every objects $x_{1}, x_{2}$ such that $x_{1}, x_{2} \in$ the carrier of $\operatorname{DualSp}(X)$ and $T\left(x_{1}\right)=T\left(x_{2}\right)$ holds $x_{1}=x_{2}$ by [26, (16), (5)], [18, (6)].

(27) Let us consider real normed spaces $X, Y$, a Lipschitzian linear operator $L$ from $X$ into $Y$, and a Lipschitzian linear operator $T$ from $\operatorname{DualSp}(X)$ into $\operatorname{DualSp}(Y)$. Suppose $L$ is isomorphism and $T$ is isomorphism and for every point $x$ of $\operatorname{DualSp}(X), T(x)=x \cdot L^{-1}$. Then there exists a Lipschitzian linear operator $S$ from $\operatorname{DualSp}(Y)$ into $\operatorname{DualSp}(X)$ such that

(i) $S$ is isomorphism, and

(ii) $S=T^{-1}$, and

(iii) for every point $y$ of $\operatorname{DualSp}(Y), S(y)=y \cdot L$.

Proof: Consider $K$ being a Lipschitzian linear operator from $Y$ into $X$ such that $K=L^{-1}$ and $K$ is isomorphism. Consider $S$ being a Lipschitzian linear operator from $\operatorname{DualSp}(Y)$ into $\operatorname{DualSp}(X)$ such that $S$ is isomorphism and for every point $y$ of $\operatorname{DualSp}(Y), S(y)=y \cdot K^{-1}$. For every 
objects $y, x, y \in$ the carrier of $\operatorname{DualSp}(Y)$ and $S(y)=x$ iff $x \in$ the carrier of $\operatorname{DualSp}(X)$ and $T(x)=y$ by [4, (5)], [29, (36)], [3, (39)], [29, (51)].

(28) Let us consider real normed spaces $X, Y$. Suppose there exists a Lipschitzian linear operator $L$ from $X$ into $Y$ such that $L$ is isomorphism. Then $X$ is reflexive if and only if $Y$ is reflexive.

(29) Let us consider a real normed space $X$. Suppose $X$ is not trivial. Then there exists a Lipschitzian linear operator $L$ from $X$ into $\Im($ BidualFunc $X$ ) such that $L$ is isomorphism. The theorem is a consequence of (10).

(30) Let us consider a real Banach space $X$. Suppose $X$ is not trivial. Then $X$ is reflexive if and only if $\operatorname{DualSp}(X)$ is reflexive.

Proof: DualSp $(X)$ is not trivial. Consider $L$ being a Lipschitzian linear operator from $X$ into $\Im($ BidualFunc $X)$ such that $L$ is isomorphism. Set $f=$ BidualFunc $X$. $\operatorname{rng} f \neq \emptyset$ by $[29$, (42)]. $\Im(f)$ is reflexive.

\section{REFERENCES}

[1] Haim Brezis. Functional Analysis, Sobolev Spaces and Partial Differential Equations. Springer, 2011.

[2] Czesław Byliński. The complex numbers. Formalized Mathematics, 1(3):507-513, 1990.

[3] Czesław Byliński. Functions and their basic properties Formalized Mathematics, 1(1): 55-65, 1990.

[4] Czesław Byliński. Functions from a set to a set Formalized Mathematics, 1(1):153-164, 1990.

[5] Czesław Byliński. Partial functions. Formalized Mathematics, 1(2):357-367, 1990.

[6] Czesław Byliński. Some basic properties of sets Formalized Mathematics, 1(1):47-53, 1990.

[7] Agata Darmochwał. The Euclidean space. Formalized Mathematics, 2(4):599-603, 1991.

[8] Peter D. Dax. Functional Analysis. Pure and Applied Mathematics: A Wiley Series of Texts, Monographs and Tracts. Wiley Interscience, 2002.

[9] Noboru Endou, Yasunari Shidama, and Katsumasa Okamura. Baire's category theorem and some spaces generated from real normed space. Formalized Mathematics, 14(4): 213-219, 2006. doi 10.2478/v10037-006-0024-x.

[10] Krzysztof Hryniewiecki. Basic properties of real numbers Formalized Mathematics, 1(1): 35-40, 1990.

[11] Jarosław Kotowicz. Convergent real sequences. Upper and lower bound of sets of real numbers. Formalized Mathematıcs, 1(3):477-481, 1990.

[12] Eugeniusz Kusak, Wojciech Leończuk, and Michał Muzalewski. Abelian groups, fields and vector spaces. Formalized Mathematics, 1(2):335-342, 1990.

[13] Kazuhisa Nakasho, Yuichi Futa, and Yasunari Shidama. Topological properties of real normed space. Formalized Mathematics, 22(3):209-223, 2014. doi:10.2478/forma-20140024

[14] Keiko Narita, Noboru Endou, and Yasunari Shidama. Dual spaces and Hahn-Banach theorem. Formalized Mathematics, 22(1):69-77, 2014. doi:10.2478/forma-2014-0007.

[15] Takava Nishivama, Keiji Ohkubo, and Yasunari Shidama. The continuous functions on normed linear spaces Formalized Mathematics, 12(3):269-275, 2004.

[16] Bogdan Nowak and Andrzej Trybulec. Hahn-Banach theorem Formalized Mathematics, 4(1):29-34, 1993.

[17] Jan Popiołek. Some properties of functions modul and signum Formalized Mathematics, $1(\mathbf{2}): 263-264,1990$. 
[18] Jan Popiołek. Real normed space Formalized Mathematics, 2(1):111-115, 1991.

[19] Michael Reed and Barry Simon. Methods of modern mathematical physics. Vol. 1. Academic Press, New York, 1972.

[20] Walter Rudin. Functional Analysis. New York, McGraw-Hill, 2nd edition, 1991.

[21] Hideki Sakurai, Hisayoshi Kunimune, and Yasunari Shidama. Uniform boundedness principle. Formalized Mathematics, 16(1):19-21, 2008. doi 10.2478/v10037-008-0003-5.

[22] Yasunari Shidama. Banach space of bounded linear operators Formalized Mathematics, 12(1):39-48, 2004.

[23] Yasumasa Suzuki, Noboru Endou, and Yasunari Shidama. Banach space of absolute summable real sequences Formalized Mathematics, 11(4):377-380, 2003.

[24] Andrzej Trybulec. On the sets inhabited by numbers. Formalized Mathematics, 11(4): 341-347, 2003.

[25] Wojciech A. Trybulec. Subspaces and cosets of subspaces in real linear space Formalized Mathematics, 1(2):297-301, 1990.

[26] Wojciech A. Trybulec. Vectors in real linear space. Formalized Mathematics, 1(2):291-296, 1990.

[27] Woiciech A. Trybulec. Subspaces of real linear space generated by one, two, or three vectors and their cosets Formalized Mathematıcs, 3(2):271-274, 1992.

[28] Zinaida Trybulec. Properties of subsets Formalized Mathematics, 1(1):67-71, 1990.

[29] Edmund Woronowicz. Relations and their basic properties. Formalized Mathematics, 1 (1):73-83, 1990. 